\title{
Lichenes ab A. Yasuda in Japonia collecti. Continuatio I.
}

\author{
Scripsit
}

\author{
Dr. Edv. A. Wainio.
}

1. Usnea molliuscula WaIN. (sp. n.).

Thallus prostratus, sat mollis, long. circ. $80 \mathrm{~mm}$, basi crass. 1-0,5 $\mathrm{mm}$, teres, cinerascenti stramineo-glaucescens, sympodialiter et dichotome ramosus, soraliis minutis aut sat minutis, crebris totus inspersus, ceterum lævigatus. Axis chondroideus tenuis. Stratum myelohyphicum laxe contextum, album, KHO supra axem vix distincte lutescens.

Prov. Harima (232). Ad corticem Bini. Ster.

2. Usnea diffracta WaIN. (sp. n.).

Thallus prostratus, fragilis, basi crass. $1-0,6 \mathrm{~mm}$, teres, dichotome et sympodialiter ramosus, stramineo-glaucescens, lævigatus, sorediis et verruculis et spinulis destitutus, transversim rimosus articulatusve, facile diffractus. Axis chondroideus tenuis. Stratum myelohyphicum crebre contextum, albidum, KHO non reagens.

Prov. Kushiro (231). Ad corticem Betula. Ster.

3. Usnea aciculifera Warn. (sp. n.).

Thallus subpendulus aut arbusculiformis, sat mollis, basi crass. 0,8-0,6 mm, teres, dichotome et sympodialiter ramosus, stramineus aut glaucescens, verruculis spinuloso-acutatis, brevissimis $(0,1-0,3 \mathrm{~mm}$ longis), et verruculis subsorediosis minutissimis maxima parte variegatim mixtimque creberrime instructus. Axis chondroideus tenuis aut sat tenuis. Stratum myelohyphicum crebre contextum, KHO lutescens fulvescensve.

Prov. Közuke (195) et Mimasaka (233). In arboribus. Ster.

4. Usnea ceratinella WaIN. (sp. n.).

Thallus prostrato arbusculiformis, sat mollis, basi crass. 1-0,8 $\mathrm{mm}$, teres, dichotome et sympodialiter ramosus, stramineo-glaucescens 
aut demum pallido-rubescens, verruculis minutis subsorediosis sat crebris et apices versus soraliis modice dilatatis et minutis crebris instructus, ramis adventitiis brevibus numerosissimis crebrisque ornatus. Axis chondroideus sat crassus. Stratum myelohyphicum crebre contextum, partim aut maculatim croceo rubescens, in apicibus albidum.

Prov. Rikuzen (230). In ramis arboris. Ster.

5. Usnea croceorubescens WaIN. (sp. n.).

Thallus arbusculiformis, sat mollis, basi crass. 1,5-1 $\mathrm{mm}$, teres, dichotome et sympodialiter ramosus, stramineo-glaucescens aut partim glaucescenti-rubescens, haud rugosus, spinulis brevissimis parcissimis aut vix ullis, verruculis minutissimis, haud distincte sorediosis, crebris instructus, at basin versus levigatus, inderdum apices versus ramis adventitiis brevibus ornatus. Axis chondroideus tenuis. Stratum myelohyphicum crebre contextum, partim croceo-rubescens.

Prov. Kushiro (234). In arbore. Ster.

6. Usnea roseola WaIN. (sp. n.).

Thallus arbusculiformis, sat mollis, basi crass. 1,5-1 mm, teres, dichotome et sympodialiter ramosus, glaucescens, haud rugosus, soraliis crebris, modice dilatatis aut partim verruculæformibus instructus, aliis verruculis vix ullis. Axis chondroideus sat tenuis. Stratum myelohyphicum crebre contextum, dilutissime roseum.

Prov. Rikuzen, Sendai (138). In arbore. Ster.

7. Usnea creberrima WaIN. (sp. n.).

Thallus asbusculiformis, rigidus, basi crass. 1,2-1 $\mathrm{mm}$, teres, haud rugosus, dichotome et sympodialiter usque ad apicem creberrime ramosus, sæpe passim etiam ramis adventitiis brevibus ornatus, verruculis crebris minutis parce subsorediosis instructus, soraliis veris destitutus, basin versus lævigatus. Axis chondroideus crassus. Stratum myelohyphicum crebre contextum, ochraceo-roseum. Ster.

Prov. Rikuzen, Yuriage (137). Ad corticem Pini Thunbergii.

8. Oropogon loxensis (FÉE) Th. Fr.

Prov. Ishikari, mons Daisetsu (225). In arbore. Ster.

9. Alectoria ochroleuca (ЕнRH.) NyL.

Thallus superne et intus $\mathrm{KHO}$ addito $\mathrm{CaCl}_{2} \mathrm{O}_{2}$ lutescens.

Prov. Ishikari, mons Daisetsu (224).

10. Ramalina intermediella WaIN. (sp. n1.).

Subsimilis $R$. subfarinaceae NYL., sed medulla KHO non reagente ab ea differens. Thallus cæspites $20-30 \mathrm{~mm}$ altos formans, dichotome sat crebre ramosus, sublinearis, compressus, solidus, laciniis basin 
versus $0,8-0,3 \mathrm{~mm}$ latis, apicem versus sensim attenuatis et filiformibus, albido-stramineis, sat lævigatis, soraliis parvis, marginalibus.

Prov. Inaba (150). Ad corticem arboris.

11. Thamnolia vermicularis (L. FIL.) ACH:

Prov. Ishikari, mons Ishikari (220).

12. Siphula cladonioidea W WIN. (sp. n.).

Thallus foliaceus, long. circ. 5, lat. 3-5 mm, sæpe convolutus, irregulariter lobatus, crass. $0,2-0,3 \mathrm{~mm}$, superne cinereo-glaucescens, inferne pallidus, KHO non reagens. S. torulosa (ThunB.) thallo subtus albido, KHO superne et inferne flavescente ab hac specie differt.

Prov. Kōzuke, mons Myōgi (142). In rupe.

13. Cetraria hiascens (Fr.) TH. Fr.

Prov. Ishikari, mons Chübetsu (214).

14. Cetraria juniperina (L.) Fr.

Prov. Shinano, mons Yatsugatake (144). Ad corticem Pini pumilæ.

15. Cetraria citrina TAYL.

Prov. Ishikari, mons Däisetsu (212).

16. Cetraria cucullata (Bell.) Ach.

Prov. Ishikari, mons Ashibetsu (213).

17. Parmelia hyporysalea WaIN. var. cinerascens WaIN.

Medulla $\mathrm{KHO}$ non reagens, $\mathrm{CaCl}_{2} \mathrm{O}_{2}$ rubescens. Thallus superne pseudocyphellis destitutus, qua nota differt a $P$. rubescente (TH. Fr.), quæ est $P$. olivetorum NyL. (haud Асн.). Thallo superne cinerascente recedit a $P$. olivetorum var. hyporysalea WaIN. (in Lich. Welw. p. 399), quæ autonoma est species.

Prov. Awaji, mons Mikuma (140 p.p.). Ad corticem arboris.

18. Parmelia inactixa (ZAHLBR.) WaIN. $P$. tinctorum var. inactiva ZABLBR. Lich. Sam. p. 50.

Thallus superne isidiosus, $\mathrm{CaCl}_{2} \mathrm{O}_{2}$ non reagens, $\mathrm{KHO}$ addito $\mathrm{CaCl}_{2} \mathrm{O}_{2}$ intus rubescens.

Prov. Nagato (190).

19. Parmelia Borreri Turn.

Thallus KHO superne flavescens, intus non reagens. Medulla $\mathrm{CaCl}_{2} \mathrm{O}_{2}$ non reagens, nequidem addito $\mathrm{KHO}$. Soralia supra laminam thalli evoluta. Huc non pertinet P. dubia (Wur.f.) Ars. Lich. Exs. n. 876.

Prov. Közuke (131). In rupe.

20. Parmelia ruderata WaIN. (sp. n.).

Thallus superne albo-punctatus isidiosusque, isidiis applanatis, 
subtus sordide albicans, ad ambitum sat anguste rhizinis destitutus, $\mathrm{KHO}$ superne demum dilute flavescens, intus non reagens, at $\mathrm{CaCl}_{2} \mathrm{O}_{2}$ rubescens. Proxima est $P$. rudectæ АсH., cujus thallus subtus fuscescenti-pallidus aut subtestaceus.

Prov. Inaba, mons Iō (145). Ad corticem arboris. Ster.

21. Parmelia isidioclada Wain. (sp. n.).

Thallus irregulariter laciniatus, margine crenulatus isidiosusque, isidiis tenuissimis brevissimisque, partim granuliformibus, laciniis 5-1 mm latis, superne cinereo-glaucescens, opacus, sat lævigatus, subtus albidus pallidusve aut medium versus obscuratus, rhizinis parcis brevibusque instructus, ambitum versus glaber, KHO superne non reagens, intus intense lutescens, deinde rubescens, $\mathrm{CaCl}_{2} \mathrm{O}_{2}$ non reagens. Affinis $P$. rudectæ AcH. et $P$. laceratulæ NyL.

Prov. Mimasaka (210). Ad corticem arboris. Ster.

22. Parmelia cetrata AcH. var. sorediifera WaIN.

Prov. Awaji, mons Mikuma (140 p.p.). Prov. Kushiro, Akkeshi (207). Ad corticem arborum. Ster.

23. Parmelia lærior NyL. Lich. Jap. p. 28.

Prov. Kōzuke (141). Ad corticem arboris. Fert.

24. Parmelia petrophila Wayn. (sp. n.).

Thallus irregulariter laciniatus, laciniis brevibus, crebris, 1,5-0,5 $\mathrm{mm}$ latis, irregulariter foveolato-inæqualibus, albido-glaucescentibus, nitidis, imbricato-confertis, laxe adpressis, subtus nigricans, rhizinis concoloribus, brevibus, sat parcis, KHO superne vix distincte flavescens, intus lutescens deindeque rubescens, sorediis et isidiis destitutus. Affinis $P$. saxatili (L.) Acr. Ster.

Prov. Kii (193). In rupe.

25. Parmelia americana (Mey. et Flot.) Mont.

Ins. Formosa (208).

26. Parmelia abstrusa WaIn. Étud. Lich. Brés. p. 64.

Ins. Formosa (209). Ad corticem arboris.

27. Parmelia conspersa (Енrh.) Ach. f. polita Flot. et f. coralloidea FLot.

Prov. Harima (191). In rupe. WaIN.

28. Parmelia pubescens (L.) WaIN. var. reticulata (Wulf.)

Prov. Kaga, mons Hakusan (135). In rupe. Fert.

29. Anzia leucobatoides (NyL.) Waln. Parmelia NyL. Lich. Nov. Zel. (1888) p. 28.

Prov. Iwashiro (152). Fert. 
30. Anzia colpota WaIN. (sp. n.).

Thallus sat crebre dichotome laciniatus, laciniis $2-0,7 \mathrm{~mm}$ latis, apice planis, ceterum convexis, albido-glaucescentibus aut apicem versus pallidis, KHO superne lutescens, intus non reagens, subtus nigricans, rhizinis parcissimis, concoloribus. Thallo crassiore, KHO superne reagente a Parmelia colpode Acr. differt.

Prov. Rikuzen, Gamō (248). Ad corticem arboris.

31. Lecanora diplotypa WaIN. (sp. n.).

Thallus modice incrassatus, areolatus, areolis angulosis, subcontiguis, circ. $0,5-0,3 \mathrm{~mm}$ latis, planis, stramineis, nudis, subnitidis, lævigatis, fere squamulæformibus, ad ambitum, haud distincte effiguratus, hypothallo indistincto. Apothecia partim crebra, adnata, basi constricta, lat. $0,4-0,9 \mathrm{~mm}$, tenuia, disco pallido vel subtestaceopallido, nudo, subnitido, lævigato, plano, margine tenui, haud prominente, stramineo, subintegro aut leviter crenulato. Hypothecium albidum, ex hyphis erectis formatum. Basis apothecii KHO demum fulvo-rubescens. Hymenium $0,050 \mathrm{~mm}$ crassum, jodo cærulescens. Epithecium testaceo-pallidum. Paraphyses arcte cohærentes, haud distincte septatæ. Sporæ 8-næ, distichæ, ellipsoideæ, apicibus rotundatis aut raro obtusis, simplices, long. 0,009-0,006, crass. 0,006-0,004 mm. Affinis est L. polytropæ NyL., ad Squamarias accedens.

Prov. Kōzuke, mons Nabewari (321). In rupe.

32. Lecanora leptocheila WAIN. (sp. n.).

Thallus tenuis, sat continuus aut minute areolato-diffractus, lævigatus, albido-cinerascens, KHO non reagens. Apothecia dispersa, emergenti-adnata, basi parum constricta, lat. 0,6-0,3 $\mathrm{mm}$, tenuia, disco fusco, nudo, opaco, plano, margine tenuissimo, cinerascente, sat integro, subpersistente, haud prominente. Hypothecium albidum. Hymenium circ. $0,120 \mathrm{~mm}$ crassum, jodo persistenter cærulescens. Paraphyses arcte cohærentes, crass. $0,001 \mathrm{~mm}$. Epithecium dilute rubricoso-rufescens. Sporæ 8-næ, distichæ, decolores, ellipsoideæ aut oblongæ, apicibus rotundatis aut obtusis, simplices, long. 0,016-0,014, crass. 0,012-0,007 mm. Affinis $L$. dispersæ NYL.

Prov. Közuke (352). In rupe.

33. Lecanora galactinula W AIN. (sp. n.).

Thallus modice incrassatus, areolatus, areolis contiguis aut partim subdispersis, circ. $0,3-0,2 \mathrm{~mm}$ latis, planis aut pr. p. convexis, angulosis aut rotundatis, albido-cinerascens, KHO non reagens. Apothecia crebra, adnata, basi leviter constricta, lat. 0,35-0,25 $\mathrm{mm}$, tenuia, disco rufo, nudo, sat opaco, plano, margine tenui, albido- 
cinerascente, integro aut minutissime crenulato, haud prominente. Hypothecium decoloratum. Hymenium jodo cærulescens. Epithecium rubricoso-rufescens. Sporæ 8-næ, subdistichæ, decolores, ellipsoideæ, apicibus rotundatis aut obtusis, simplices, long. 0,009-0,007, crass. 0,005-0,004 mm. L. galactiniza NyL. (Свомв. Lich. Cap. p. 173) huic speciei habitu est similis, at thallo KHO flavescente et apotheciis 0,3-0,5 mm latis ab ea differt, affinis $L$. campestri (Schær.). $L$. galactinella NyL. -(l.c.) apotheciis a thallo parum prominentibus et margine integro a planta nostra distinguitur.

Prov. Közuke (358). In rupe.

34. Lecanora rufoalbida W AIN. (sp. n.).

Thallus modice incrassatus, areolatus, areolis contiguis aut parce subdispersis, $0,6-0,2 \mathrm{~mm}$ latis, angulosis aut rotundatis, leviter convexis, albido-cinerascentibus, lævigatis, parum nitidis, KHO non reagentibus, hypothallo nigricante limitatus. Apothecia sparsa et partim crebra, basi constricta, tenuia, lat. $0,9-0,5 \mathrm{~mm}$, disco rufo, nudo, opaco, margine tenui, albido, subintegro, haud prominente. Sporæ decolores, ellipsoideæ, simplices, long. 0,012-0,014, crass. 0,006 mm (sec. Yasudam). Apotheciis et sporis majoribus differt a $L$. galactinula WAIN.

Prov. Közuke (333). In rupe.

35. Lecanora aggregans W

Thallus areolatus, areolis dispersis, $0,5-0,2 \mathrm{~mm}$ latis, sat crassis, leviter convexis, fere verrucæformibus, albido-cinerascentibus aut cinereis, KHO non reagentibus, hypothallo obscurato parum evoluto. Apothecia lat. $0,2-0,3 \mathrm{~mm}$, sæpe aggregata contiguaque, acervulis sparsis, tenuissima, basi parum constricta, sæpe flexuosa, disco rufescente aut testacea, nudo, margine tenuissimo, subintegro. Hypothecium pallidum. Epithecium pallidum, KHO non reagens. Paraphyses crass. 0,0005 $\mathrm{mm}$, increbre ramoso-connexæ. Asci clavati, long. circ. 0,070, crass. $0,018 \mathrm{~mm}$. Sporæ 8-næ, monostichæ aut distichæ, decolores, simplices, ellipsoideæ, apicibus rotundatis, long. $0,010-0,006$, crass. $0,006-0,0045$ $\mathrm{mm}$. Gonidia pleurococcacea.

Prov. Kōzuke, mons Nabewari (356). In rupe.

36. Lecanora glaucodeella WaIN. (sp. n.).

Thallus sat tenuis, verruculosus, verruculis $0,1-0,15 \mathrm{~mm}$ latis, convexis, sparsis et partim contiguis, partim farinoso-sorediosus, stramineus aut stramineo-glaucescens, hypothallo indistincto. Apothecia lat. $0,9-0,6 \mathrm{~mm}$, sparsa, modice incrassata, basi constricta, disco livido-pallescente, tenuiter pruinoso, margine modice incrassato, 
rotundato convexo, integro aut verruculoso, thallo concolore. Hypothecium albidum. Hymenium circ. $0,1 \mathrm{~mm}$ crassum, jodo persistenter cærulescens. Epithecium dilutissime subrufescens. Paraphyses arcte cohærentes, crass. $0,0015 \mathrm{~mm}$, apice leviter incrassatæ, increbre septatæ. Sporæ 8-næ, subdistichæ, decolores, simplices, ellipsoideæ, apicibus rotundatis, long. 0,010-0,008, crass. $0,007-0,005 \mathrm{~mm}$. Affinis $L$. glaucolutescenti NyL. et L. glaucodeæ NYL.

Prov. Kōzuke (354). In rupe.

37. Lecanora conspersa WalN. (sp. n.).

Thallus modice incrassatus, areolatus, areolis contiguis aut dispersis, vulgo leviter aut demum bene convexis, flavido-stramineis, lævigatis, subnitidis, esorediatis, hypothallo indistincto. Apothecia lat. 0,7-0,5 mm, sparsa, tenuia, basi leviter constricta, disco plano, testaceo aut rufescenti-testaceo, nudo, subnitido, lævigato, margine tenui, leviter crenulato, haud prominente, thallo concolore. Epithecium rufescens. Sporæ 8-næ, decolores, simplices, ellipsoideæ, apicibus rotundatis, long. $0,010-0,009$, crass. $0,007-0,005 \mathrm{~mm}$. Affinis $L$. perspersæ NyL. et L. chlorophæodi NyL.

Prov. Kōzuke (339). In rupe.

38. Lecanora pseudisterella WaIN. (sp. n.).

Thallus crassus aut modice incrassatus, continuus aut areolatus aut demum rimoso-diffractus, verruculoso-inæqualis, albido-cinerascens vel subalbidus, sat opacus, KHO flavescens. Apothecia lat. 0,7-0,4 $\mathrm{mm}$, primum subimmersa, demum emergentia et basi levissime constricta, tenuia, disco plano aut leviter convexo, rufo, nudo, opaco, lævigato, margine tenui, haud prominente, subintegro, albido. Нypothecium albidum. Hymenium jodo persistenter cærulescens. Epithecium rufescens. Sporæ 8-11æ, distichæ, decolores, simplices, ellipsoideæ, apicibus rotundatis, long. 0,010-0,007 $(-0,013 \mathrm{~mm}$, observante YASUdA), crass. 0,007-0,005 mm. Proxima est L. galactinizz NyL. (Сromв. Lich. Cap. p. 173), quæ apotheciis adhuc minoribus et thallo tenuiore ab ea differt. $L$. pseudistera NyL. apotheciis paullo majoribus ab his recedit, at minoribus quam ia L. campestri (Sch ER.) instructa est. L. expansa (Асн.) sec. herb. Асн. apotheciis fere sicut in L. pseudistera NyL. et ab ea vix distinguenda.

Prov. Iwashiro, mons Iide (318). In rupe.

39. Lecanora subimmergens WAIN. (sp. n.).

Thallus modice incrassatus, lævigatus, areolato-diffractus, areolis minutis, albido-cinerascens, opacus, KHO lutescens, dein fulvescens, hypothallo indistincto. Apothecia lat. $0,8-0,5 \mathrm{~mm}$, diu immersa, 
demum leviter emergentia, basi haud constricta, disco plano aut raro demum convexo, rufo, nudo, leviter nitido, lærigato, margine integro aut raro flexuoso, haud prominente, tenui. Hypothecium pallidum. Hymenium circ. $0,070 \mathrm{~mm}$ crassum, jodo persistenter cærulescens. Epithecium rufescens. Paraphyses arcte cohærentes, crass. 0,0015 $\mathrm{mm}$, apice haud incrassatæ. Sporæ 8-næ, decolores, simplices, ellipsoideæ, apicibus rotundatis, long. circ. $0,007-0,006$, crass. 0,006-0,005 mm. Thallo lævigato et apotheciis minus emergentibus a specie præcedente differt.

Prov. Kōzuke (355). In rupe.

40. Lecanora gallifera Wain. (sp. n.).

Thallus modice incrassatus, continuus aut diffractus, verruculosoinæqualis, albido-cinerascens, KHO non reagens, sæpe tuberculis hemisphæricis 3,5-0,6 mm latis, morbosis sparsis instructus, hypothallo indistincto. Apothecia lat. 2-1,2 mm, elevata, basi bene constricta, disco plano, rufescente, nudo, opaco, margine sat crasso, sublobulato crenato, modice prominente. Hypothecium albidum, a hymenio haud distincte limitatum. Paraphyses arcte cohærentes, apice leviter incrassatæ. Sporæ 8-næ, distichæ aut monostichæ, decolores, simplices, ellipsoideæ, apicibus rotundatis, long. 0,016-0,010, crass. 0,007-0,005 $\mathrm{mm}$. Habitu inter $L$. intumescentem et $L$. allophanam intermedia est.

Prov. Kōzuke, mons Akagi (346). Ad corticem arboris.

41. Lecanora cinereocarnea (Eschw.) WaIn.

Prov. Kii (34.9). Ad corticem arboris.

42. Lecanora atra (Huds.) AcH.

Prov. Kōzuke, mons Akagi (323). Ad corticem arboris.

43. Lecanora (Aspicilia) decorata WaIn. (sp. n.).

Thallus crassus (1-6 $\mathrm{mm})$, inæqualiter incrassatus, ceterum lævigatus, diffractus, stramineus, partim demum obscuratus, $\mathrm{KHO}+$ $\mathrm{CaCl}_{2} \mathrm{O}_{2}$ superne lutescens, intus jodo non reagens. Apothecia lat. $0,7-4 \mathrm{~mm}$, thallo immersa aspicilioideaque aut raro demum emergentia et margine thallino basi constricto instructa, disco nigro, nudo, nitido, haud impresso. Hypothecium stramineo-pallidum albidumve. Hymenium crassit. 0,080-0,110 mm, jodo cærulescens. Epithecium cæruleoæruginosum (KHO sordide olivaceum aut olivaceo-smaragdulum), strato gelatinoso $0,010-0,006 \mathrm{~mm}$ crasso decolore lævigatoque obductum. Paraphyses arcte cohærentes, simplices, crass. 0,0015 mm, apice capitatæ $0,004-0,005 \mathrm{~mm}$ crassæ. Sporæ 8-næ, distichæ, 
decolores, simplices, ellipsoideæ apicibusque rotundatis aut subglobosæ, long. 0,011-0,015, crass. 0,006-0,009 $\mathrm{mm}$.

Prov. Shinano, mons Yatsugatake (130). In rupe.

44. Lecanora cinerea (L.) Sommerf.

Prov. Közuke, mons Nabewari (347). In rupe.

45. Lecanora gibbosa (Aсr.) NyL.

Prov. Kōzuke (322). In rupe.

46. Lecanora calcarea (L.) Sommerf. var. Hoffmanni (Ach.) SOMMERF.

Prov. Közuke $(257,303)$. In rupe.

47. Lecanora phymatodisous Wain. (sp. n.).

Thallus modice incrassatus, lævigatus, crebre areolato-rimosus, cervinus vel pallide cinereo-fuscescens, opacus, nec KHO nec jodo reagens, hypothallo indistincto. Apothecia thallo immersa, disco 0,3-0,2 $\mathrm{mm}$ lato, subanguloso difformive, impresso, nigro, opaco, nudo, verruca depressa instructo. Hypothecium albidum. Hymenium jodo cærulescens. Epithecium rufescens. Paraphyses crass. 0,001 $\mathrm{mm}$, crebre septatæ, parce ramoso-connexæ, gelatinam abundantem percurrentes. Sporæ 8-næ, distichæ, ellipsoideæ, decolores, simplices, long. 0,020-0,024, crass. $0,009-0,010 \mathrm{~mm}$. L. griseolam TH. FR. in memoriam revocans.

Prov. Kōzuke, mons Akagi (320). In rupe.

48. Lecanora coiliodes WaIN. (sp. n.).

Thallus modice incrassatus, verruculoso-inæqualis aut verrucosoareolatus, partim subdispersus, stramineus, opacus, KHO non reagens, hypothallo indistincto. Apothecia verrucis immersa, disco $0,2-0,5 \mathrm{~mm}$ lato, bene impresso, atro, nudo. Hypothecium albidum. Hymenium 0,050-0,055 mm crassum, jodo cærulescens. Epithecium olivaceum, KHO rufescens. Paraphyses arcte cohærentes, crass. $0,001 \mathrm{~mm}$, apice $0,003 \mathrm{~mm}$ crebre septatæ, in KHO laxe cohærentes. Sporæ distichæ, decolores, ellipsoideæ, simplices, long. circ. 0,016, crass. $0,010 \mathrm{~mm}$. Affinitate incerta.

Prov. Kōzuke (353). In rupe.

49. Placopsis pacifica WAIN. (sp. n.).

Thallus sat lævigatus, ad ambitum breviter laciniatus, medium versus late sorediosus, soraliis confluentibus, haud prominentibus, soraliis erasis demum leproso-verruculosus. Cum Pl. gelida ab auctoribus nonnullis commixta. Etiam e Nova Zelandia (a L. Lindsay lecta) in herb. NyL. sub hoc nomine adest.

Prov. Iwashiro, mons Bandai (164). In rupe. 
50. Ochrolechia pallescens NyL. (variatio aut autonoma species).

$\mathrm{KHO}$ addito $\mathrm{CaCl}_{2} \mathrm{O}_{2}$ thallus superne rubescens et intus lutescens, margo apothecii superne et intus rubescens, discus rubescens. $\mathrm{CaCl}_{2} \mathrm{O}_{2}$ solo non reagentes. Nimis parce lecta.

Prov. Kōzuke, mons Nabewari (236). In rupe.

51. Ochrolechia orientalis WarN. (sp. n.).

Margine apotheciorum extus verrucoso differt ab $O$. upsaliensi (L.), quæ reactionibus ei est similis. Planta in Lich. Yasun. Jap. p. 2 hoc nomine determinata ad $O$. orientalem pertinet.

Prov. Hōki, mons Daisen (99). Ad corticem arboris.

52. Ochrolechia Yasudæ WaIN. Lich. Yasud. Jap. (1918) p. 2.

Thallus et margo et discus apotheciorum nec $\mathrm{KHO}$ nec $\mathrm{CaCl}_{2} \mathrm{O}$ reagens. Thallus isidiis crebris, brevibus, partim verrucæformibus. Margine apotheciorum transversim striato aut sat lævigato.

Prov. Kōzuke, mons Akagi (156 p.p.). Ad corticem arboris.

53. Ochrolechia akagiensis YASUdA in litt.

Thallus modice incrassatus, verruculoso-inæqualis, albido-cinerascens, $\mathrm{KHO}$ addito $\mathrm{CaCl}_{2} \mathrm{O}_{2}$ lutescens maculis parvis rubris. Margo et discus apotheciorum his reagentiis rubescens, $\mathrm{CaCl}_{2} \mathrm{O}_{2}$ dilute rubescens. Apothecia lat. $1,5-1 \mathrm{~mm}$, disco pallido, pruinoso, margine modice incrassato, prominente, sat lævigato. Sporæ observante cel. YASUDA long. 0,065-0,067, crass. 0,020-0,022 mm.

Prov. Kōzuke, mons Akagi (156 a). Supra muscos destructos.

54. Ochrolechia diademata WaIN. (sp. n.).

Thallus sat crassus, albido-cinerascens, isidiis crebris, brevibus, $0,15-0,25 \mathrm{~mm}$ crassis, partim verrucæformibus instructus, sorediis destitutus, KHO addito $\mathrm{CaCl}_{2} \mathrm{O}_{2}$ rubescens. Margo et discus apotheciorum eodem modo reagentes. $\mathrm{KHO}$ et $\mathrm{CaCl}_{2} \mathrm{O}_{2}$ solo haud reagentes. Apothecia lat. 1,5-1. $\mathrm{mm}$, disco albido-pruinoso, margine bene prominente, crebre abundanterque verruculoso. Affinis $O$ akagiensi et $O$. Yasudæ quibuscum crescit.

Prov. Kōzuke, mons Akagi $(156$ b). Ad corticem vetustum arboris.

55. Pertusaria (Lecanorastrum) flayosulphurea WaIN. (sp. n.).

Thallus modice incrassatus, leviter verruculoso-inæqualis, stramineoflavescens (intus albus), opacus, $\mathrm{KHO}$ non reagens, at addito $\mathrm{CaCl}_{2} \mathrm{O}$ rubescens, soraliis prominentibus, $0,5 \mathrm{~mm}$ latis, albis.

Prov. Harima, mons Hokke (174). In rupe.

56. Pertusaria perfida NłL. Lich. Nov. Zel. (1888) p. 69. 
Thallus et discus KHO non reagens (etiam in orig. NYL.).

Prov. Kōzuke (360). In rupe.

57. Pertusaria subfallens WaIN. (sp. n.).

Thallus modice incrassatus, lævigatus aut partim rugosus, cinereoglaucescens, leviter nitidus, $\mathrm{KHO}$ addito $\mathrm{CaCl}_{2} \mathrm{O}_{2}$ intus lutescens. Pseudostromata $\mathrm{KHO}$ addito $\mathrm{CaCl}_{2} \mathrm{O}_{2}$ extus et intus lutescentia, apothecia solitaria aut raro plura continentia. $P$. commutatæ et $P$. variolosæ subsimilis.

Prov. Kōzuke, mons Akagi (294, 297). Ad corticem arborum.

58. Pertusaria velatula WaIN. (sp. n.).

Thallus modice incrassatus, sat lævigatus aut inæqualis, albidoglaucescens aut cincrascens, parum nitidus, KHO non reagens, at addito $\mathrm{CaCl}_{2} \mathrm{O}_{2}$ lutescens et demum rubescens. Pseudostromata apothecia solitaria continentia, $\mathrm{KHO}$ non reagentia, at addito $\mathrm{CaCl}_{2} \mathrm{O}_{2}$ intus lutescentia ct demum rubescentia. Sporæ solitariæ, oblongæ, long. $0,140-0,160$, crass. $0,035-0,045 \mathrm{~mm}$, membrana haud striata (observante cel. YASUDA). Affinis $P$. velatæ.

Prov. Kōzuke, mons Akagi (295). Ad corticem arboris.

59. Pertusaria yaginatula WaIN. (sp. n.).

Thallus modice incrassatus, sat lævigatus, cinerascens, nec jodo, nec $\mathrm{KHO}$, nec $\mathrm{CaCl}_{2} \mathrm{O}_{2}$ reagens. Pseudostromata apothecia solitaria aut pauca continentia, intus $\mathrm{KHO}$ addito $\mathrm{CaCl}_{2} \mathrm{O}_{2}$ leviter rubescentia. Affinis $P$. subvaginatæ NyL.

Prov. Közuke, mons Akagi (299). Ad corticem arboris.

60. Pertusaria leucosora NYL.

Prov. Kōzuke, mons Nábewari (301). In rupe.

61. Pertusaria (Porophora) pustulata (AсH.) NyL.

Prov. Inaba (176). Ad corticem arboris.

62. Pertusaria stenostoma Wain. (sp. n.).

Thallus tenuis, sat lævigatus aut leviter verruculoso-inæqualis, albido-cinerascens, nec $\mathrm{KHO}$ nec $\mathrm{CaCl}_{2} \mathrm{O}_{2}$ reagens. Pseudostromata sat crebra, verrucas formantia subhemisphæricas aut difformes, 0,5$1,2 \mathrm{~mm}$ latas, basin versus leviter dilatatas aut sat abruptas, apothecia solitaria continentia aut rarius 2-3 confluentia, nec KHO nec $\mathrm{CaCl}_{2} \mathrm{O}_{2}$ reagentia, disco leviter impresso, nigricante, angulosorotundato aut sæpe oblongo difformive, lat. $0,1-0,15$, long. 1-0,1 mm. Sporæ 8-næ-2-næ, oblongæ, long. 0,064-0,080, crass. 0,026 $\mathrm{mm}$, membrana $0,003-0,004 \mathrm{~mm}$ crassa, haud striata. Facie externa $P$. pustulatam in memoriam revocans.

Prov. Hōki, mons Daisen (173). Ad corticem arboris, 
63. Pertusaria læyis Knıght ; Müll. Arg. Lich. Beitr. n. 771, Consp. Lich. Nov. Zel. p. 54.

Prov. Kōzuke (277). Ad corticem arboris.

64. Pertusaria conformis Warn. (sp. n.).

Sporis 8-nis, sec. YasudaM 0,160-0,200 mm longis, 0,050-0,062 $\mathrm{mm}$ crassis, membrana haud striata differt a $P$. subobductante NyL., Lich. Jap. p. 54. Thallus et pseudostromata extus haud reagentia, intus lutescentia deindeque (præsertim zona gonidiali) subrubescente vel aurantiaco-rubescentia. Habitu $P$. leioplacx similis est.

Prov. Kōzuke (286). Ad corticem arboris.

65. Pertusaria corallina (L.) ARN.

Prov. Inaba (134). In rupe.

66. Pertusaria cinereoobscurata WaIN. (sp. n.).

Thallus crassus aut sat crassus (1-0,2 mm), inæqualis, obscure cinereus, nec $\mathrm{KHO}$ nec $\mathrm{CaCl}_{2} \mathrm{O}_{2}$ reagens. Pseudostromata valde difformia, tuberculosa, 1-6 $\mathrm{mm}$ lata, $0,5-2,5 \mathrm{~mm}$ alta, inæqualia, aut parum conspicua et vix prominentia, intus demum leviter lutescentia et maculis dilutissime sordide rubescentibus, apothecia numerosa aut solitaria continentia, verrucis leviter prominentibus indicata, disco inconspicuo. Sporæ sec. cel. YASUdAM 4-næ, lung. 0,115-0,175, crass. 0,043-0,060 mm, membrana lævigata. Ad sect. Irregulares MüLL. ARG. pertinet.

Prov. Kōzuke, mons Akagi (283). Ad corticem arboris.

67. Pertusaria conferta Wain. (sp. n.).

Thallus modice incrassatus aut sat ,crassus, leviter inæqualis, cinerascens aut sordide albicans, KHO zona gonidiali partim rubescente, parum visibilis, pseudostromatis crebris instructus. Pseudostromata tuberculiformia, 1-0,2(-2) $\mathrm{mm}$ lata, irregularia, crebra contiguaque aut subconfluentia, lateribus sæpe præruptis, raro basi leviter constricta, zona gonidiali KHO partim rubescente. Sporæ "binæ, long. 0.130-0,165, crass. 0,030-0,045 mm, membrana lævigata" (sec. cel. YASUDAM). $P$. subobductans NYL. var. cinereoglauca WAIN. reactione thalli ab ea differt.

Prov. Kōzuke $(287,291,292)$. Ad corticem arborum. N. 283 ad formam obscurierem WAIN., thallo intense cinereo instructam, pertinet.

68. Pertusaria petrophyes Knight, Contr. Lich. N.S. Wales p. 47 ; Nyl. Fl. 1886 p. 326 ; Wain. React. Lich. Müll. p. 9.

Var. hokiensis WAIN. Thallus et pseudostromata albido-cinerascentia, KHO non reagentia. Sporæ (observante cel. YASUDA) long. 
0,060-0,078, crass. 0,026-0,032 mm, membrana lævigata. Pseudostromata partim contigua.

Prov. Hōki (180). In rupe.

69. Pertusaria inabensis WaIN. (sp. n.).

Subsimilis $P$. nagasakensi NyL., at thallo et pseudostromatibus KHO extus impure fulvescentibus, intus lutescentibus et sporis paullo majoribus, sec. cel. Yasudam long. circ. 0,128, crass. $0,058 \mathrm{~mm}$, membrana lævigata. Thallus crassus, substrato laxe affixus. Pseudostromata irregularia, ostiolis impressis.

Prov. Inaba (285).

70. Pertusaria rugulosa WaIN. (sp. n.).

Thallus sat tenuis, lævigatus, partim verruculis increbre inspersus, albidus, KHO demum maculis rubescentibus, intus non reagens. Pseudostromata sat dispersa, sat depressa, 1-2 $\mathrm{mm}$ lata, 1-5 apothecia continentia, lateribus præruptis, ostiolis nigris, parvis, dispersis, marginibus ostiolaribus vulgo impressis, KHO extus demum rubescentia, intus non reagentia. Sporæ binæ, long. 0,150-0,110, crass. 0,040-0,030 mm, membrana costulata. $P$. subrugosa NyL., Lich. Jap. p. 52, huic habitu est similis, ostiolis lutescentibus indistinctis et reactione thalli ab ea differens.

Prov. Kōzuke, mons Akagi (289). Ad corticem arboris.

71. Pertusaria Yasudm Wain. (sp. n.).

Thallus modice incrassatus, verrucoso-inæqualis, albidus, KHO non reagens. Pseudostromata depresse hemisphærica, lateribus præruptis, apice impresso, in depressione difformi cinereo-nigricante ostiola 7-1 continentia, ostiolis confertis, punctiformibus, nigris, partim contigua aut confluentia, sat lævigata aut ambitu leviter verrucosa, KHO non reagentia. Sporæ " binæ, long. 0,100-0,135, crass. 0,030-0,040 mm, membrana lævigata" (sec. cel. YASUDAM). Subsimilis $P$. leucodi KNight (NyL. Lich. Nov. Zel. p. 71), quæ sporis ab ea differt.

Prov. Hōki (177 a). Ad corticem arboris.

72. Pertusaria commixta Warn. (sp. n.).

Thallus modice incrassatus, verrucoso-inæqualis, albidus, $\mathrm{KHO}$ in zona gonidiali levissime subrubescens. Pseudostromata irregulariter verrucosa, difformia, contigua et confluentia, lateribus præruptis aut basi leviter insculptis, apice leviter impresso, in depressione difformi dilute cinereo nigricante ostiola solitaria aut pauca continentia, zona gonidiali K'HO rubescente. Sporæ "binæ, long. 0,114-0,130, crass. 0,030-0,034 mm, membrana lævigata" (sec. cel. YASUdAM). Affinis speciei præcedenti. 
[Vol. $\operatorname{xxxV}$. No. 411.

Prov. Inaba $(177$ b). Ad corticem arboris.

73. Pertusaria kurohiensis WaIN. Lich. Jap. (1918) p. 3.

Sporæ binæ, long. 0,140-0,200, crass. 0,040-0,050 mm, membrana lævigata (sec. cel. Yasudam). Proxime affinis $P$. diffidenti Nyl., Lich. Jap. p. 52.

Prov. Kōzuke, mons Akagi (281). Prov. Mutsu, mons Hakkōda (179). Ad corticem arborum.

74. Pertusaria akagiensis WaIN. (sp. n.).

Thallus sat crassus, crebre plicato- et subverrucoso-inæqualis, cinerascens, KHO superne non reagens, intus lutescens et demum fulvo-rubescens. Pseudostromata partim crebra, tuberculiformia, lat. 5-1 $\mathrm{mm}$, basi leviter insculpta, apothecia numerosa continentia, intus lutescentia deindeque fulvo-rubescentia, extus maculis dilute rubescentibus, ostiolis haud aut parum impressis, margine ostiolari annulum parum distinctum formante. Sporæ 4-næ, long. 0,180-0,270, crass. 0,050-0,060 mm, membrana lævigata. Affinis $P$. kotsukensi WaIN. (Lich. Jap. p. 3), quæ vix nisi reactionibus ab ea differt.

Prov. Kozuke, mons Akagi $(280,284)$. Ad corticem arborum.

75. Pertusaria sublæxiganda $\mathrm{W}_{\text {AIN. }}$ (sp. n.).

Thallus sat lævigatus aut leviter verruculis depressis increbre inspersus, cinereo-glaucescens, KHO zona gonidiali demum rubescente. Pseudostromata dispersa, lat. 1-3,5 mm, sæpe applanata, difformia, lateribus præruptis, ad zonam gonidialem KHO demum rubescentia, apothecia numerosa continentia, margine ostiolari parum prominente, ostiolo minuto, sæpe foveolato. Sporæ " 8-næ, long. 0,058-0,094, crass. 0,022-0,028 $\mathrm{mm}$, membrana lævigata" (sec. cel. YASUdAM). Reactionibus differta $P$. læviganda NyL. Lich. Jap. p. 53.

Prov. Kōzuke, mons Akagi (288). Ad corticem arboris.

Var. pauperior WaIN.

Sporæ binæ, long. 0,115-0,145, crass. 0,035-0,040 mm, membrana lævigata (sec. cel. YASUdAM).

Prov. Kōzuke, mons Akagi (290). Ad corticem arboris.

76. Perforaria epileiodes Wain. (sp. n.).

Thallus tenuis, verruculis minutis inspersus, cinerascens, KHO non reagens. Apothecia sat crebra, subglobosa, lat. $0,6-0,8 \mathrm{~mm}$, basi insculpta, apice leviter impresso, depressione plano, albido-pallido, cinereo-glaucescenti-limbato. Perithecium albidum, globosum, jodo cærulescens, in depressione apothecii denudatum, ceterum amphithecio thallino, gonidia continente, extus cinerascente obductum, ostiolo minutissimo. Paraphyses simplices, crass. $0,0008 \mathrm{~mm}$, glatinam 
abundantem percurrentes. Sporæ 8-næ-2-næ, distichæ, decolores, ellipsoidex aut oblongx, long. $0,056-0,064$, crass. $0,024-0,030 \mathrm{~mm}$, membrana $0,002 \mathrm{~mm}$ crassa, lævigata. Affinis Perforariæ epileiæ (NyL. Lich. Jap. p. 57), quæ cephalodiis numerosis, apotheciis majoribus et thallo crassiore ab ea differt.

Prov. Harima, mons Hokke (169). Ad corticem arboris.

77. Pilophorus Hallii (Tuck.) WaIN. (Pilophoron clavatum NyL. Lich. Jap. p. 17).

Prov. Kōzuke (276). In rupe.

78. Placodium granulosum (Schæer.) WaIN.

Thallo sorediis instructo a P1. elegante differens.

Prov. Közuke, mons Mitsumine (143). In rupe.

79. Placodium pyropœcilum (NyL.) WAIN. (Lecanora pyropœcila NyL. in Cromb. Lich. Cap., 1877, p. 172).

Thallus areolatus (etiam in specimine orig.), dispersus, haud granulosus. Affine P1. Mülleri WaIN.

Prov. Kōzuke (312). In rupe.

80. Placodium fumosum WaIN. (sp. n.).

Thallus sat tenuis, crustaceus, areolato-diffractus areolatusve, areolis contiguis, cinereis, sat lævigatis. Apothecia tota basi substrato adnata, haud constricta, lat. 0,4-0,25 $\mathrm{mm}$, tenuia, quasi inter areolas thalli sita et eas parum superantia, disco vitellino aut sordide vitellino-rubescente, nudo, plano, margine tenuissimo, nigricante, discum haud superante. Sporæ haud evolutæ. Affine P1. benguellensi NyL. et P1. Janino WaIN.

Prov. Közuke, mons Nabewari (338). In rupe.

81. Placodium gilyofumosum WaIN. (sp. n.).

Thallus tenuis, crustaceus, sat continuus, leviter verruculosoinæqualis, impure albidus aut subcinerascens. Apothecia sat crebre, basi bene constricta, lat. $0,5-0,3 \mathrm{~mm}$, crass. circ. $0,180 \mathrm{~mm}$, disco plano, vitellino-rubescente, nudo, margine modice incrassato, prominente, integro, cinerascente aut raro cæruleo-nigricante. Excipulum gonidia continens. Epithecium fulvescens, KHO purpureum. Sporæ 8-næ, orculæformes, apicibus obtusis, decolores, long. 0,011-0,014, crass. 0,006-0,007 mm. Affine Placodio Bœrgesenii WalN.

Prov. Kōzuke (341). In rupe.

82. Placodium ferrugineum (Huds.) HePP.

Prov. Inaba, mons Sensoku (151 p.p., cum Anaptychia hypochræa). Ad corticem arboris.

83. Anaptychia hypochræa WaIN. (sp. n.). 
Thallus laciniis circ. 10-15 $\mathrm{mm}$ longis, 2-0,2 $\mathrm{mm}$ latis, adscendentibus, laxe imbricatis, superne albido-cinerascentibus, KHO non reagentibus, subtus decorticatis, ochraceis et $\mathrm{KHO}$ violascentibus, margine fibrilloso, fibrillis simplicibus aut parce ramosis, subalbidis. Affinis $A$. podocarpx.

Prov. Inaba, mons Sensoku (151). Ad corticem arboris. Ster.

84. Anaptychia hypoleuca (Murlens.) Wain. var. Schæreri HEPP. Sorediis destituta.

Prov. Rikuzen, Shōbuta (364). Ad corticem Pini densifloræ.

85. Anaptyohia heterochroa WaIN. (sp. n.).

Thallus iteratim dichotome ramosus, ramis laxe adpressis, circ. 1-0,5 mm latis, superne albidis, KHO vix distincte flavescentibus, intus lutescentibus, subtus decorticatus, ochraceus et KHO purpureus, fibrillis marginalibus, nigris, simplicibus et demum squarroso-ramosissimis et soraliis marginalibus instructus. Affinis $A$. hypoleucæ.

Prov. Kōzuke (229). Ad corticem Pini densifloræ. Ster.

86. Physoia glaucovirescens NyL. (Syn. Lich. p. 419, Fl. 1869 p. 322).

Var. tenebricosa WAIN.

Thallus subtus nigricans, rhizinis nigricantibus.

Prov. Nagato (256). In rupe.

87. Physcia trichophora Hue (Lich. Extra-eur. n. 348).

Var. cinereoalbida WAIN.

Thallus cinereo-albicans, KHO non reagers, subtus sordide vel pallide albicans, rhizinis brevibus, long. $0,5-0,2 \mathrm{~mm}$, increbris. Excipulum glabrum.

Prov. Inaba (45, 153). Ad corticem arboris.

88. Physcia imbricata Wain. (sp. n.).

Thallus foliaceo-squamosus, KHO non reagens, squamis varie confertis, long. 5-1, lat. 1,5-0,5 mm, adpressis, demum marginem versus sorediosis, subcuneatis, lobatis vel irregulariter laciniatis, superne glauco-cinerascentibus aut partim cervinis, subtus nigricantibus, rhizinis nigricantibus, brevibus. Apothecia excipulo subtus trichoso, disco nigricante. Affinis $\mathrm{Ph}$. cycloseli AcH.

Prov. Rikuzen, Shiogama (269). Ad corticem Pini densifloræ.

89. Melanaspicilia fumosa Wain. (sp. n.).

Thallus crustaceus, areolatus aut rimoso-diffractus, areolis 2-0,5 mm latis, angulosis, lævigatis, modice incrassatis, cinereis, opacis, intus KHO flavescentibus. Apothecia sat crebra, aspicilioidea, areolis immersa, immarginata, disco nigro, nudo, plano, thallum haud super- 
ante. Hypothecium fusconigrum. Epithecium fusconigrum. Sporæ 8-næ, fuscescentes, ellipsoideæ, 1-septatæ, septo sat tenui, membrana æqualiter incrassata, long. circ. 0,017 , crass. 0,009 $\mathrm{mm}$. Medulla jodo hand cærulescens.

Prov. Közuke (329). In rupe.

90. Melanaspicilia kozukensis WAIN. . (sp. n.).

Thallus crustaceus, sat tenuis, areolatus, areolis adnatis, vulgo contiguis, angulosis, $0,3-0,5 \mathrm{~mm}$ latis, cinereo-pallidis aut albidocinerascentibus, KHO non reagentibus, medulla non cærulescente, hypothallo nigricante parce conspicuo. Apothecia dispersa, areolis immersa aut raro demum leviter emergentia, immarginata aut raro demum margine thallino, integro, tenui, thallum parum superante instructa, disco 0,4-0,2 $\mathrm{mm}$ lato, nigro, nudo, plano. Hypothecium pallidum. Hymenium superius sat dilute rufescens. Sporæ 8-næ, fuscescentes, ellipsoideæ, 1-septatæ, haud constrictæ, long. circ. 0,0170,018 , crass. $0,010-0,012 \mathrm{~mm}$, membrana sat inæqualiter incrassata. Habitu subsimilis Lecanoræ confragosulæ Nyı. (in Cromb. Lich. Cap. p. 172), quæ thallo areolato-squamuloso, areolis hypothallo nigro instratis, ab ea differt. Etiam Rinodina ocellata hanc in memoriam revocans.

Prov. Közuke (315). In rupe.

91. Melanaspicilia crebra WaIN. (sp. n.).

Thailus sat tenuis, crustaceus, areolatus, areolis $0,5-0,2 \mathrm{~mm}$ latis, angulosis, contiguis, planis, lævigatis, cinereo-fuscescentibus, opacis, nec KHO nec jodo reagentibus, hypothallo indistincto. Apothecia crebra, aspicilioidea, areolis immersa, immarginata, disco 0,3-0,2 $\mathrm{mm}$ lato, nigro, nudo, plano, thallum haud superante. Hypothecium albidum. Epithecium fuscescens. Sporæ 8-næ, distichæ, nigricantes, ellipsoideæ, 1-septatæ, septo tenui, membrana æqualiter modice incrassata, long. 0,010-0,012, crass. 0,005-0,007 $\mathrm{mm}$.

Prov. Közuke (317). In rupe.

92. Rinodina cervina WAIN. (sp. n.).

Thallus sat tenuis aut modice incrassatus, crustaceus, areolatus, areolis 1-0,2 mm latis, angulosis, planis, sat lævigatis aut levissime verruculoso-inæqualibus, cervino-luridis vel cinereo-fuscescentibus, opacis, $\mathrm{KHO}$ non reagentibus, hypothallo indistincto. Apothecia partim sat crebra, thallo adnata, basi leviter constricta, 0,3-0,4. mm lata, margine thallino, tenui, integro, discum haud superante, disco plano, fusco-nigricante aut partim pallido-rufescente, nudo. Hypothecium decoloratum. Paraphyses apice clavatæ fuscescentesque aut raro 
pallidiores. Excipulum jodo non. reagens. Sporæ 8-næ, nigricantes, ellipsoideæ, 1-septatæ, long. 0,014-0,017, crass. 0,007 mm, septo tenui, membrana sat æqualiter incrassata. $R$. milvinæ affinis.

Prov. Közuke (359). In rupe.

93. Rinodina subalbida (NyL.) W AIN. Lecanora sophodes AcH. ${ }^{*}$ L. subalbida Nyı. Lich. Jap. p. 40.

Excipulum jodo non reagens. Sporæ long. 0,017-0,024, crass. 0,009-0,011 mm, 1-septatæ, membrana inæqualiter incrassata, septo tenui aut sat tenui aut raro crasso et poro instructa. Apothecia margine integro, albido. Thallus verrucosus, albidus, KHO non reagens.

Prov. Kōzuke (325). Ad corticem arboris. Collegit K. Tsunoda.

94. Rinodina akagiensis WAIN. (sp. n.).

Thallus sat tenuis, crustaceus, areolatus, areolis dispersis, 0,5-0,3 $\mathrm{mm}$ latis, planis aut depressis, fuscescentibus aut cinereo-fuscescentibus, hypothallo indistincto. Apothecia dispersa, lat. 1,2-0,8 mm, adnata, basi constricta, disco plano aut rarius demum depresso-convexo, fusco aut fusco-nigricante, nudo, margine tenui aut sat tenui, integro, cinereo-fuscescente cinerascenteve. Excipulum jodo haud reagens. $\mathrm{Hy}$ pothecium albidum. Epithecium fuscescens aut rubricoso-pallidum, KHO non reagens. Sporæ 8-næ, distichæ, intense nigræ, ellipsoideæ aut oblongæ, apicibus obtusis, rectæ aut curvatæ, raro leviter constrictæ, long. 0,030-0,034, crass. 0,012-0,016 mm (sec. cel. Yasudam long. $0,034-0,040$, crass. $0,016-0,018 \mathrm{~mm})$, membrana inæqualiter incrassata, septo tenui. Facie externa $R$. homobolam (NyL.) in memoriam revocans.

Prov. Kōzuke, mons Akagi (348). Ad corticem arboris.

95. Buellia Yasudæ WaIN. Lich. Jap. (1918) p. 4.

Thallus KHO demum leviter flavescens. Sporæ long. 0,011, crass. 0,004 mm, 3-septatx.

Prov. Awaji (310). Ad corticem arboris. Collegit S. MatsuZAWA. 